\title{
Transparenz und Geheimnis
}

\section{Timon Beyes und Claus Pias}

Stellt der Transparenzbegriff eine Leitvokabel der Gegenwart dar, so bezeichnet die Digitalisierung die medientechnische Bedingung seiner Ubiquität. Im Ideal und in der Ideologie der Transparenz hat sich, so Manfred Schneider, in den letzten gut zwanzig Jahren ein "messianisches Potential« verdichtet (Schneider 2013: 13). Das entspricht ungefähr der Zeitspanne, in der sich eine Form der digitalen Welterzeugung durchgesetzt hat, deren technologische Zurüstung die Systeme und Prozesse von Kommunikation, Wahrnehmung und Sinngebung prägt. Diese Entwicklung legt nahe, von Digitalen Kulturen zu sprechen - im Plural, denn der Heterogenität dieser sozio-technischen Gefüge scheinen unterschiedliche Formen der Welterzeugung zu entsprechen, die mit der die Lebenswelt durchdringenden digitalen Medienumwelt entstehen.

Dem Messianismus gläserner, geheimnisfreier Sphären von Politik und Wirtschaft, der von den Glasfaserkabeln des Internets und der Allgegenwärtigkeit intelligenter und teils ohne Umweg über Subjekte kommunizierender Artefakte zehrt, wird der Albtraum einer `Transparenzgesellschaft entgegengestellt, in der das entblößte Leben der Einzelnen zu Big Data in den Händen von Netzkonzernen und staatlichen Überwachungsagenturen wird, die - ihrerseits intransparent - sammeln und auswerten, was digitale Nutzer an Spuren hinterlassen (Han 2012). Aktivisten und Aktivistinnen wiederum experimentieren mit medientechnisch ermöglichten
Taktiken der Intransparenz und Geheimhaltung, um der nutzerbezogenen Repräsentation von Identität in die Anonymität oder in wechselnde temporäre Subjektpositionen zu entkommen - mit Anonymous als bis dato markantestem Beispiel. Und nicht zuletzt bestätigt die Snowden/NSA-Affäre Schneiders lakonisches Diktum: »Transparenz hier und jetzt gibt es nicht (Schneider 2013: 14).

Gleichzeitig rückt der Geheimnisverrat Snowdens den im gegenwärtigen, moralisch aufgeladenen Diskurs zu Wohl und Wehe des digitalen Transparenzbegehrens vernachlässigten Begriff des Geheimnisses in den Mittelpunkt. Unser Debattenbeitrag und die auf ihn folgenden Kommentare sind dem Versuch gewidmet, Digitale Kulturen nicht, oder nicht primär, in Potential und Problematik der Transparenz (und korrespondierender Leitbegriffe wie Partizipation und Öffentlichkeit) zu denken, sondern im Zeichen des Geheimen, der fundamentalen Intransparenz und des Arkanums. Dabei ist im ersten Schritt kurz an die allgemeine soziale Form des Geheimnisses und deren Funktionalität jenseits ihrer - zumindest gemäß Alltagsverständnis - ethischen Bedenklichkeit zu erinnern, die einen nüchternen Blick auf das Verbergen und Verraten ermöglicht. Im zweiten, ausführlicheren Schritt möchten wir einen experimentell-historischen $\mathrm{Zu}$ gang wagen, der nahelegt, die Gegenwart Digitaler Kulturen aufgrund ihrer zeitlichen Struktur unter Rückgriff auf vormoderne Geheimnistypen neu 
$\mathrm{zu}$ beleuchten. Unser Vorschlag umfasst also eine doppelte Wendung: einerseits die Frage danach, wie Digitale Kulturen vom Geheimnis aus gedacht werden können, andererseits aber, ob unsere gegenwärtigen Konzepte und Begriffe des Geheimen sich überhaupt für dieses Denken als tragfähig erweisen können.

Wer über die soziale Form des Geheimnisses nachdenkt, kommt zunächst an Georg Simmels mäandernden Ausführungen über »Das Geheimnis und die geheime Gesellschaft" nicht vorbei. Unabhängig ihrer Inhalte und damit verknüpfter Wertzuschreibungen bezeichnen die "Attraktionen des Geheimnisses" (Simmel 1999: 409) für Simmel einen notwendigen Tatbestand der Differenziertheit sozialer Verhältnisse, die durch die Differenziertheit genauso ermöglicht sind wie sie sie verschärfen. Das Geheimnis,

"das durch negative oder positive Mittel getragene Verbergen von Wirklichkeiten, ist eine der größten Errungenschaften der Menschheit; gegenüber dem kindischen Zustand, in dem jede Vorstellung sofort ausgesprochen wird, jedes Unternehmen allen Blicken zugängig ist, wird durch das Geheimnis eine ungeheure Erweiterung des Lebens erreicht, weil vielerlei Inhalte desselben bei völliger Publizität überhaupt nicht auftauchen könnten« (Simmel 1999: 406).

Die Geschichte der Geheimhaltung zu schreiben heißt somit, Gesellschaftsentwicklung nachzuzeichnen: eine Abfolge von Offenbarem, das zum Geheimnis wird, und von Geheimnissen, die sich offenbaren. Es entsteht eine Art Nullsummenspiel von $\mathrm{Zu}$ - und Abfluss geheimniswürdiger Inhalte, von Verbergen und Enthüllen, Heimlichkeit und Verrat, mit dem Geheimnis als geheimnisvollem Operator gesellschaftlicher Evolution.
Nun lässt sich Simmels abschließende Spekulation, dass »die Angelegenheiten der Allgemeinheit immer öffentlicher, die der Individuen immer sekreter würden « (Simmel 1999: 411), mit Blick auf die Gegenwart Digitaler Kulturen in Frage stellen: Weist nicht die Selbstentblößung digitaler Nutzer im Netz auf die Porosität der Unterscheidung zwischen öffentlich und privat hin, und zeigt das Aufdecken verborgener Datenmengen durch WikiLeaks und Snowden nicht trotz oder durch ihre Veröffentlichung die Existenz enormer Geheimnisapparate? Uns scheinen eher die folgenden grundlegenden Lehren Simmels fruchtbar zu sein: Das Geheimnis ist als fundamentale Kategorie der Kulturanalyse ernst zu nehmen. Es bedarf einer historischen Untersuchung unterschiedlicher Formen der Geheimhaltung, um über gegenwärtige Spielarten nachzudenken (Assmann/Assmann 1997ff.). Und es ist angezeigt, das Geheimnis in historischer Perspektive gewissermaßen anders herum zu denken: Die Frage ist nicht, was geheim gehalten wird, sondern was überhaupt verraten werden kann, und was - indem es zum Objekt des Verrats werden kann oder nicht - den Stellenwert und die Logik des Geheimnisses in verschiedenen Kulturen und zu verschiedenen Zeiten ausmacht (Horn 2007).

In diesem Sinne möchten wir das Gedankenexperiment vorschlagen, Digitale Kulturen jenseits von Konzepten einer Moderne zu denken, deren letztes Produkt Digitale Kulturen zwar selbst sind, die diese Moderne aber möglicherweise bereits beendet haben (Lyotard 1986). Vielleicht machen viele der gegenwärtig leidenschaftlich geführten Debatten diesen Umstand gerade durch einen latenten Anachronismus deutlich, der sich durch die Verwendung etablierter Konzepte wie Transparenz, Öffentlichkeit und Partizipation äußert (Baxmann/ Beyes/Pias 2014). Was aber wäre, wenn man diesen Anachronismus verschärfen und versuchen würde, Digitale Kulturen gerade nicht mehr durch moderne 
(oder immer noch darauf bezogene postmoderne), sondern durch die Fremdheit vormoderner Konzepte zu verstehen? Denn dort besitzt insbesondere das Geheimnis eine andere, fruchtbar zu machende historische Semantik.

So zog noch bis zum 17. Jahrhundert die Kosmologie eine Grenze zu einem wesensmäßig Geheimen, die das Außen eines fundamental Unbekannten in Form natürlicher Geheimnisse markierte. Erst die neuzeitliche Wissenschaft legte Einspruch gegen solche Geheimnisse ein, und zwar mit dem Ziel, mit natürlichen Mitteln Erkenntnis über die Natur zu erlangen und die »kosmisch-religiöse Stoppregel « zu entmachten (Luhmann/Fuchs 1989: 104). Zeit geriet damit zur Möglichkeit, natürliche Geheimnisse zu entparadoxieren, wie man systemtheoretisch sagen würde. In vergleichbarer Weise galten auch die shohen Dinge des Staates ihrer Natur nach als geheim. Hier sind die Entschlüsse, Entscheidungen und Taten des Herrschers von Weisheit als einer geheimen und prinzipiell unergründbaren Intelligenz getragen, ohne die die Stabilität des Staates nicht zu gewährleisten wäre. Sie liegen offen zutage, aber ihr Grund «kann « nicht verraten und damit auch nicht diskutiert werden. Sie sind (in kosmologischer Analogie) inkommunikabel wie alle großen Dinge der Natur und darum nicht allein Weisheit, deren Willkür »gegen Trivialität geschützt, also geheim bleiben muß" (Luhmann/Fuchs 1989: 116), sondern ein strukturell > unverratbares` Geheimnis.

In diesem Kontext war die Behandlung des Geheimnisses wohl differenzierter als heute (oder in Simmels Skizze), weil die Vormoderne verschiedene Geheimnistypen kennt, die anderen Begriffen, Methoden und Rationalitäten folgen, wie etwa die arcana cordis, die arcana dei, die arcana mundi oder die arcana imperii. Im Sinne unseres Arguments gilt es dabei vor allem die Unterscheidung zwischen mysterium (als dem Nicht-Wissbaren und damit NichtVerratbaren) und secretum (als einem Verborgenen, das intelligibel gemacht und dadurch verraten werden kann) mitzuführen. So schließen die arcana imperii beides ein: das mysterium der Weisheit und Willkür des Herrschers als Zentrum eines unverratbaren Grundes und zugleich das Gewimmel einer Vielzahl kleiner oder größerer secreta, die Gegenstand des Verrats und der Geheimhaltung gegen alle Arten von intelligence (also wörtlich: der prinzipiell möglichen Einsicht) sind.

Demgegenüber operieren unsere gegenwärtig in den Feuilletons und der Politik geführten Debatten zu Datenschutz und Persönlichkeitsrechten mit einer anderen, aus dieser Sicht stark reduzierten Spielart von Geheimnissen, nämlich denen, die man verraten kann. Sobald man sich in dieser modernen Kategorie bewegt, lässt sich ein Geheimnis nur noch entweder verraten oder nicht verraten, offenlegen oder geheimhalten. Ohne diese Hegemonie eines bestimmten Geheimnistyps kann der Transparenzgedanke sogenannter »bürgerlicher Öffentlichkeit» nicht zur Entfaltung kommen. Es ist das Geheimnis, das man freilegen kann und muss, und es ist zugleich das Geheimnis, bei dem nicht klar ist, ob der Staat Angst vor seinen Bürgern haben muss oder umgekehrt. Mit diesem Suspektwerden von Souveränität und einem aktiven Demaskierungsinteresse an Arkana scheint der Typus des unverratbaren Geheimnisses untergegangen bzw. in andere Bereiche ausgelagert worden zu sein.

Das unverratbare Geheimnis wird stattdessen, wie Koselleck gezeigt hat, in einer neuen Zeitordnung aufgehoben (Kosellek 1989). Die Moderne hat gewissermaßen das unverratbare Geheimnis der Souveränität in die Zeit verlegt. Fortan ist die Zukunft ein Geheimnis - aber eben ein Geheimnis, das nicht verraten werden kann. Und sie hat die Frage der Zukunft unverbrüchlich mit der Vorstellung von Öffentlichkeit und Partizipation verbunden. Beide sind eingebettet in einen Zusammenhang von Geheimnis und Transparenz, der sich auf Zukunft 
hin ausrichtet. Sonst wäre Partizipation - so unser modernes, davon immer schon geprägtes Verständnis - sinnlos, denn sie spielt sich zwischen Sein und Sollen ab: zwischen dem wie die Welt >ist $<$ und wie sie (anders) sein ıkönnter. In diesem Sinne hat das 18. Jahrhundert bekanntlich eine neue Form von Geschichtlichkeit und damit die Geschichte selbst erfunden. Und sie hat die Gegenwart zum entscheidenden Ort zwischen »Erfahrungsraum « und »Erwartungshorizont « gemacht (Kosellek 1989: 349375), zum Austragungsort einer prinzipiell `offenen Zukunft.

Die These, die wir im Hinblick auf Digitale Kulturen vorschlagen möchten, lautet nun, dass sich eine neue Zeitordnung etabliert - ein "Chronotop" (Hans Ulrich Gumbrecht), der sich von der in der Sattelzeit 1780-1830 entstandenen und unser Denken bestimmenden Zeitordnung unterscheidet. Diese Veränderung hat, so unsere Vermutung, mit der Entstehung der modernen Kybernetik nach 1945 begonnen. Mit den Konzepten von Feedback und selbstregulierenden Systemen, von Prädiktion und Digitalcomputern hat ein grundlegender Umbau von Zeitstrukturen begonnen, wie Norbert Wiener bereits 1948 vermutet hat (Wiener 1992: 63-81). Mit der Digitalisierung weiter Teile unserer Lebenswelt, mit der schier endlosen Zahl von Apparaten, die ohne den Umweg über Subjekte miteinander kommunizieren und sich, im Größten wie im Kleinsten, gegenseitig steuern und Feedback geben, haben sich diese besonderen, kybernetischen Zeitverhältnisse gewissermaßen verabsolutiert. Sie erzeugen eine Zeitordnung, in der moderne Geschichtlichkeit kollabiert. In Anlehnung an Robert Musil könnte man dies vielleicht einen »Absolutismus der Gegenwart» nennen, oder mit Hans Ulrich Gumbrecht als »breite Gegenwart« beschreiben (Gumbrecht 2010). Der kybernetische Chronotop Digitaler Kulturen stellt daher die Frage geschichtlicher Zeiten noch einmal zur Diskussion.
Diese Diagnose ist nicht neu. Einen ähnlichen Deutungsversuch unternahm bereits Vilém Flusser vor knapp 25 Jahren (Flusser 1991). Wenn es, so Flusser, tatsächlich einen Verbund von kybernetischen Maschinen gibt, die sich durch Feedback verschalten, die sich adaptiv verhalten und Störungen selbständig verarbeiten, und die durch das, was heute als Big Data zusammengefasst wird, die Datenspuren von Subjekten mit der Prädiktion von Subjektivierungsformen zusammenfallen lassen dann kollabiert das Verhältnis von Sein und Sollen und damit das moderne Konzept von Zukunft. Diesen Zustand hat Flusser (wie andere Denker vor und nach ihm) als »Posthistoire« bezeichnet, als »Nachgeschichte«. Innerhalb dieser neuen Zeitordnung kann es, so seine Diagnose, aus logischen Gründen kein Argument, keine Kritik und kein Politisches mehr geben. Und darum ist Partizipation, so folgert Flusser radikal, »Nonsens «. ${ }^{1}$ Diesem düsteren Versuch stellen wir einen experimentell-historischen gegenüber. Er lautet, die Gegenwart Digitaler Kulturen gerade nicht in modernen, sondern versuchsweise in vormodernen Begriffen zu denken. Denn wenn die moderne Zeitordnung tatsächlich problematisch geworden oder gar kollabiert ist, bestünde die Herausforderung darin, Digitale Kulturen nicht länger in Kategorien der Transparenz, Öffentlichkeit und Partizipation zu denken, sondern im Zeichen einer fundamentalen Intransparenz, im Zeichen des Arkanums.

Wenn nun für die Entstehung dieser neuen Zeitordnung die kybernetischen Konzepte von Feedback und selbstregulierenden Systemen, von Prädiktion und Digitalcomputern aufgerufen werden, dann konstatieren wir zugleich, dass digitale, vernetzte Medien das Agens dieses Chronotops sind. Die Alltagsbeispiele sind zahllos: So sind mittlerweile ganze Industrien mit der Prädiktion beschäftigt, welche Musik man gerne hören oder welche Serie man gerne schauen möchte, mit wem man 
eigentlich befreundet sein müsste, oder wie man den Stau auf dem Weg zur Arbeit umfährt. Je größer die Datenmengen, desto größer die Wahrscheinlichkeit, auch den scheinbar unberechenbarsten Hakenschlag des Subjekts vorherzusagen, wie einst den Zickzack-Kurs eines ausweichenden Feindflugzeugs. Man entkommt sich selbst nicht mehr, sondern begegnet sich im Gegenteil immer nur selbst und seiner überraschenden Erwartbarkeit. Solche Weisen des Zukunftsverlusts, solches Verwischen von Erfahrungsraum und Erwartungshorizont zugunsten einer medientechnischen Rückkopplung von Vergangenheit und Zukunft lässt sich möglicherweise mit vormodernen Zeitkonzepten besser in den Blick nehmen.

Demzufolge ließe sich auch die These aufstellen, dass die like-Kultur sogenannter Social Media weniger mit moderner Partizipation zu tun hat als vielmehr mit vormodernen Ritualen. Likes scheinen eher den états, cortes, Reichs- oder Landestagen zu ähneln, wie sie vom Spätmittelalter bis ins 18. Jahrhundert üblich waren. Solche »Partizipations«-Formen, von denen aus kein direkter Weg in die Moderne führt, waren Rituale des Konsens und nicht Aushandlungen eines Dissens - und genau dieses Ritualhafte hat die Moderne als Gegenteil des Politischen desavouiert. Sie folgten einer Logik, die mit einer auf Argumenten und Transparenz aufgebauten Partizipation einer teilhabenden Öffentlichkeit nichts $\mathrm{zu}$ tun hat. Sie waren notwendige, performative Teilnahmeformen innerhalb einer nicht zukunftsgerichteten Zeitordnung, weil ihnen das Konzept der Entscheidung fehlte (Krischer 2010).

Diese leicht vermehrbaren Beispiele werfen die Frage auf, wie viel man über die »Apparate« (Flusser) wissen kann und muss, die diese neue Eigenzeit Digitaler Kulturen herstellen, was ihr Geheimnis sein könnte, das es vielleicht transparent zu machen gälte und welche Geheimnisse sie bergen, die es zu veröffentlichen oder zu schützen gälte. Insofern würde dieser Deutungsversuch mit der Frage nach dem »Verstehen « von digitalen Medien stehen oder fallen. Und dieses "Medien-Verstehen " ist möglicherweise ein melancholisches Unternehmen, wie Friedrich Kittler bereits vor dreißig Jahren vermutete. Seine berühmte Beschreibung, wie Medien »unsere Lage bestimmen" geschieht in einem Moment, in dem sich bereits die Grenze ihrer Unmöglichkeit angesichts der Verwirklichung Digitaler Kulturen abzeichnet. Oder mit Kittlers eigenen Worten: »In der allgemeinen Digitalisierung [...] verschwinden die Unterschiede zwischen den einzelnen Medien. [...] ein totaler Medienverbund auf Digitalbasis wird den Begriff Medium selber kassieren. Statt Techniken an Leute anzuschließen, läuft das absolute Wissen als Endlosschleife« (Kittler 1986: 7f.).

Der seitdem laut werdende Ruf nach neuen Repräsentationsversuchen, gar neuen Poetiken netzwerkbasierter Steuerung und Kontrolle (Galloway 2011) kann somit als Versuch verstanden werden, der Unsichtbarkeit, Unrepräsentierbarkeit und Inkommensurabilität von Algorithmen ein anderes "Verstehen" von digitalen Medien entgegenzusetzen und auf den Absolutismus der Gegenwart mit neuen Bildern und Denkformen zu antworten, die über den Verrat von secreta à la Snowden hinausgehen und dem Mysterium eines medientechnisch bedingten arcanum gerechter werden. Dies betrifft insbesondere die daran anschließende Frage des Handelns und der Handlungsgrundlagen, wenn diese nicht mehr im Horizont eines transparenzbestimmten, argumentierfähigen und zukunftsoffenen Chronotops stattfinden. Der Transparenzdiskurs, der stets eine ethische Forderung reklamiert oder reflektiert, gerät an die Grenzen eines Geheimnisses, das dazu herausfordert, eine Ethik ohne Transparenz und eine Zukunft ohne das überkommene Verständnis von Partizipation und Öffentlichkeit (und seine Kritikformen) neu zu denken (Latour 2007; Foerster 2008). 
Ein Blick auf die Klimaforschung ist in diesem Zusammenhang besonders schlagend, denn kaum eine Wissensdomäne ist epistemologisch so abhängig vom historischen Stand der Hard- und Software: einerseits von beobachtbaren Qualitätssprüngen durch schiere Rechenleistung, andererseits aber von einer Softwaregeschichte, in deren schlecht oder gar nicht dokumentierten Millionen Code-Zeilen sich archäologische Schichten wissenschaftlichen Denkens sedimentiert haben, die aus gutem Grund nicht berührt oder umgeschrieben, sondern nur erweitert, global standardisiert und zertifiziert werden. Was in den Supercomputern eines solchen globalen Forschungsverbundes vorgeht, ist - auch für die beteiligten Wissenschaftler - gar nicht mehr transparent zu machen. Und an dem, was sich daraus an alternativen Welten (und nicht etwa Prognosen) ergibt, was unser Handeln und unsere Selbstwahrnehmung leiten soll, versagen zugleich die Routinen der Kritik. Der gängige Reflex, die `Konstruiertheit des Wissens aufzuweisen, verschlägt wenig, denn er erspart nicht das Handeln angesichts von Szenarien, die sich ihres eigenen Konstruktivismus sehr wohl bewusst sind. Und die Falsifizierbarkeit der klassischen Wissenschaftsethik ist (nicht bloß aus kapazitären, sondern auch aus systematischen Gründen) nicht praktizierbar, weil der Gegenstand des Klimas nicht experimentalisierbar ist.

Einige seriöse Klimaforscher fordern daher inzwischen eine neue Kosmologie, um unser $\mathrm{Zu}$ kunftshandeln global zu begründen. Ist diese Forderung auf alle möglichen Felder auszuweiten, in denen der Grad der Vernetzung, der Rechenleistung und der Softwareentwicklung ein Maß an Komple- xität erreicht haben, an dem kein Verstehen und kein Nachvollzug mehr möglich ist, die Ergebnisse aber dennoch ein Feedback von Gegenwart und Zukunft herstellen? Wenn sich nun, wie in diesem Beispiel, das Politische um ein Zentrum des NichtVerstehens rankt, kann es kein modernes Transparenzkonzept des Wissens mehr geben, keine Partizipation durch Mitsprache »in der Sache« (Schelsky 1965). Und auch dies kann man vormodern, unter Rückgriff auf das Denken des Geheimnisses ausdrücken: Die Legitimationsstrategie computersimulierter Klima-Kosmologie gehört ins vormoderne politische Register der Souveränität. Klimaforschung ist gleichsam eine neue science royale. An die Stelle, die einst die Weisheit (oder Willkür) des Herrschers bezeichnete, eine Stelle, die durch eine metaphysische Erkenntnisgrenze geschützt war, rückt damit die Datenverarbeitung. Die Souveränität der Datenverarbeitung zieht erneut eine Grenze zu etwas, was sich konstitutiv entzieht, weil es seiner »Natur « nach geheim ist. Nur dass es nicht mehr Natur und nicht mehr Kosmologie ist, sondern Technik. Das nannte man früher, in Bezug auf souveräne Herrschaft, einmal Arkanum.

1 In der Folge entwickelt Flusser dann die Figuren des Funktionärs, des Verzweifelten, des Terroristen, des Technokraten und des Umweltschützers als Sozialtypen einer Gegenwart, in der Partizipation logisch unmöglich geworden ist. 


\section{Literatur}

Assmann, Aleida/Assmann, Jan (1997-1999): Schleier und Schwelle, München: Fink (Bd. 1: »Geheimnis und Öffentlichkeit«, Bd. 2: »Geheimnis und Offenbarung«, Bd. 3: »Geheimnis und Neugierde»).

Baxmann, Inge/Beyes, Timon/Pias, Claus (Hg.) (2014): Soziale Medien - Neue Massen, Zürich: diaphanes.

Flusser, Vilém (1991): Gesten. Versuch einer Phänomenologie, Düsseldorf: Bollmann.

Foerster, Heinz von (2008): KybernEthik, Berlin: Merve.

Galloway, Alexander (2011): »Are some things unrepresentable?«. In: Theory, Culture \& Society 28 (7-8), 85-102.

Gumbrecht, Hans Ulrich (2010): Unsere breite Gegenwart, Frankfurt a.M.: Suhrkamp.

Han, Byung-Chul (2012): Transparenzgesellschaft, Berlin: Matthes \& Seitz.

Horn, Eva (2007): Der geheime Krieg: Verrat, Spionage und moderne Fiktion, Frankfurt a.M.: Fischer.

KitTler, Friedrich (1986): Grammophon - Film - Typewriter, Berlin: Brinkmann \& Bose.

Koselleck, Reinhard (1989): Vergangene Zukunft. Zur Semantik geschichtlicher Zeiten, Frankfurt a.M.: Suhrkamp.

Krischer, André (2010): »Das Problem des Entscheidens in systematischer und historischer Perspektive«. In: Herstellung und Darstellung von Entscheidungen. Verfahren, Verwalten und Verhandeln in der Vormoderne, hg. v. André Krischer/Barbara StollbergRilinger, Berlin: Duncker \& Humblot (Zeitschrift für Historische Forschung, Beiheft 44), 35-64.
Latour, Bruno (2007): Elend der Kritik: Vom Krieg um Fakten zu Dingen von Belang, Zürich: diaphanes.

Luhmann, Niklas/Fuchs, Peter (1989): Reden und Schweigen, Frankfurt a.M.: Suhrkamp.

LyotaRd, Jean François (1986): Das postmoderne Wissen: Ein Bericht, Graz/Wien: Passagen.

Schelsky, Helmut (1965): »Der Mensch in der wissenschaftlichen Zivilisation«. In: Ders.: Auf der Suche nach Wirklichkeit. Gesammelte Aufsätze, Düsseldorf/ Köln: Diederichs, 439-480.

SCHneIder, Manfred (2013): Transparenztraum. Literatur, Politik, Medien und das Unmögliche, Berlin: Matthes \& Seitz.

SimmeL, Georg (1999): Soziologie. Untersuchungen über die Formen der Vergesellschaftung [1908], Gesamtausgabe Bd. 11, hg. v. Otthein Rammstedt, 3. Aufl., Frankfurt a.M.: Suhrkamp.

Wiener, Norbert (1992): Kybernetik. Regelung und Nachrichtenübertragung im Lebewesen und in der Maschine, Düsseldorf/Wien: Econ. 